\title{
UNIFORM ASYMPTOTIC APPROXIMATIONS TO THE SOLUTIONS OF THE DUNN-LIN EQUATIONS*
}

\author{
BY \\ B. S. NG \\ Department of Mathematical Sciences \\ Indiana University-Purdue University \\ Indianapolis, Indiana \\ AND \\ Indianapolis Center for Advanced Research \\ Indianapolis, Indiana
}

\begin{abstract}
In this paper, we develop a uniform asymptotic theory for the Dunn-Lin equations which govern the stability of compressible boundary layers at moderate Mach numbers. "First approximations" to the solutions are derived when the Prandtl number is equal to unity, in which case the structure of the approximations is especially simple. The rapidly varying parts of the approximations can be expressed in terms of certain generalized Airy functions and the slowly varying parts can be expressed in terms of quantities all but one of which are well-known from the older heuristic theories. The approximations obtained in this way are uniformly valid in a full neighborhood of the turning point. Because of the simplicity of the present theory, it is expected that the techniques developed in this paper can also be applied to other more general stability equations for compressible boundary layers, such as the Lees-Lin equations.
\end{abstract}

1. Introduction. The asymptotic theory for higher-order differential equations with a turning point has been greatly advanced in recent years through work on the Orr-Sommerfeld equation which governs the stability of incompressible parallel shear flows. There is also a closely related class of problems which arises from the study of the stability of boundary layers in a compressible fluid. These equations-of which the Dunn-Lin system to be discussed in this work is an example-are higher-order systems with a simple turning point. Historically, the asymptotic theories for these equations were direct generalizations of the heuristic techniques developed for the Orr-Sommerfeld equation [6,10], and thus they share much of the same limitations. The most serious difficulty is the lack of uniformity of the approximations obtained, which leads to considerable controversy as to the validity of their use in the subsequent formulation of characteristic equations. Despite these facts, heuristic methods have proved to be adequate for the incompressible case. The corresponding situation concerning compress-

* Received April 20, 1974. The author is most grateful to Professor William H. Reid for suggesting this problem, and for his helpful advice in the course of research. He is also grateful to Dr. D. W. Dunn and Professor W. D. Lakin for many helpful comments. The work reported in this paper has been supported in part by the National Science Foundation under grants GP-8620 and GP-20426, and was completed while the author was a postdoctoral fellow in the Department of Mathematics at the University of Toronto. 
ible boundary layers is, however, less satisfactory. It should be remarked that all the numerical calculations based on the existing asymptotic theories have failed to produce correct curves of neutral stability. The failure of the older asymptotic methods has not been fully understood, and it is of considerable interest, therefore, to re-examine the problem in light of the recent developments relating to the Orr-Sommerfeld equation.

In the present work, uniform "first approximations" have been derived for the Dunn-Lin equations, the least complex of all the governing systems proposed for the study of compressible boundary layer stability. Moreover, lest the present theory be obscured by unnecessary technical complexities, we have restricted our attention to the case where the Prandtl number is equal to unity. The present approach is closely related to some methods developed by Olver [9] whereby he obtained uniform approximations to a certain class of second-order equations, and can be regarded as a direct extension of the work of Reid [12,13] on the Orr-Sommerfeld equation to higher-order systems with a simple turning point. The techniques employed here also represent a complete departure from the usual procedure of seeking approximations in terms of viscous and non-viscous types (see, for example, [1] and [14]). Within the framework of the uniform theory, such a distinction can no longer be maintained, and it has been found to be more appropriate to characterize the approximations by their asymptotic properties together with certain matching conditions. In the course of studying the Dunn-Lin equations by the method of matched asymptotic expansions, it became clear that when the Prandtl number is one, the inner expansions can be expressed to all orders in terms of the class of generalized Airy functions first defined by Reid [11]. This suggests that we seek uniform approximations to the solutions which exhibit precisely the same symmetries in the complex plane as the generalized Airy functions. The structure of these approximations can then be inferred directly from the inner expansions and by the use of certain recursion formulae which the special functions satisfy. These approximations consist of rapidly varving parts which can immediately be expressed in terms of the generalized Airy functions, and slowly varying coefficients which can be derived if we require that they be regular at the turning point and that the approximations themselves satisfy the Dunn-Lin equations. In this respect, the present theory bears certain resemblances to the well-known comparison equation method (see, for example, [7]). But the need of what would in our case be a "comparison system" has been eliminated, as we seek uniform approximations asymptotically in terms of the generalized Airy functions instead of the solutions of a suitably chosen "comparison system." Moreover, the procedure for determining the slowly varying coefficients can be substantially simplified by matching the uniform approximations to their outer expansions within their respective domains of validity. The approximations thus obtained are uniformly valid in a bounded domain containing the turning point.

Finally, a remark on the use of the Dunn-Lin equations in the present analysis is in order. Because of the various assumptions and approximations made in the course of their derivation (cf. [2]), these equations must be regarded as a first approximation to the other more complete governing systems which have been proposed, such as the Lees-Lin equations [4]. It is generally believed that they become inaccurate for high Mach numbers, since terms which enter into the second approximation then become relatively more important and may not always be negligible. In fact, the inherent inaccuracy of the Dunn-Lin equations has often been considered as one of the sources of difficulties in obtaining neutral curves from them for high Mach numbers. Never- 
theless, an attempt by Lees and Reshotko [5] to develop an asymptotic theory based on an augmented set of equations also unexpectedly ran into similar difficulties. Subsequently, it was determined through the numerical work of Mack [8] that both the Dunn-Lin and the Lees-Reshotko asymptotic theories fail to produce accurate curves of neutral stability for Mach numbers greater than 1.6, a value for which the Dunn-Lin equations are expected to be adequate. Hence, aside from the question of accuracy of the governing equations themselves in describing the physical phenomena, there appear to be some fundamental difficulties which are mathematical in nature. In this connection, the Dunn-Lin equations seem to be most appropriate for the purpose of the present paper since, despite their relative simplicity, they clearly exhibit all of the essential mathematical features of the problem. A satisfactory uniform asymptotic theory for the Dunn-Lin equations can therefore be expected to clarify the corresponding difficulties for the other stability equations of compressible boundary layers in general.

2. The governing equations. The Dunn-Lin equations were first proposed by Dunn and Lin [2] in an attempt to deal with the stability of compressible boundary-layers with respect to three-dimensional disturbances in terms of a two-dimensional problem. They began by considering the complete system of linearized partial differential equations for three-dimensional disturbances and their asymptotic properties for large Reynolds numbers. By a consistent approximation scheme based on an order-of-magnitude analysis of all the terms involved, they were able to derive a set of simplified equations which, they suggested, would be adequate in a first approximation. An important feature of the simplified system is the absence of the dissipation term from the energy equation, and it is mainly because of this simplification that Dunn and Lin were then able to perform a Squire-type transformation on the equations. ${ }^{1}$ For a three-dimensional disturbance of the form

$$
q^{\prime}(x, y, z, t)=q(y) \exp \{i(\alpha x+\beta z-\alpha c t)\},
$$

where $\alpha, \beta$ are wave numbers and $c$ is the complex wave speed, a rotation of the coordinate system in the $(x, z)$ plane through an angle $\psi=\tan ^{-1}(\beta / \alpha)$ leads to an uncoupling of the $z$-momentum equation and a simultaneous elimination of the $z$-dependence in the other equations. The resulting system of equations for the amplitude functions of the disturbances are generally known as the Dunn-Lin equations, and in non-dimensional form they are given by

$$
\begin{gathered}
\rho\left\{i \alpha(U-c) f+U^{\prime} \alpha \phi\right\}=\frac{\mu}{R} f^{\prime \prime}-\frac{i \alpha}{\tau M^{2}} \pi, \\
\rho\left\{i \alpha^{2}(U-c) \phi\right\}=\frac{\mu}{R} \alpha \phi^{\prime \prime}-\frac{1}{\tau M^{2}} \pi^{\prime}, \\
\rho\left\{i \alpha(U-c) \theta+T^{\prime} \alpha \phi\right\}=\frac{\mu}{\sigma R} \theta^{\prime \prime}+\frac{\tau-1}{\tau}\{i \alpha(U-c) \pi\}, \\
i(U-c) r+\rho^{\prime} \phi+\rho\left(i f+\phi^{\prime}\right)=0, \quad \pi=\frac{r}{\rho}+\frac{\theta}{T},
\end{gathered}
$$

${ }^{1}$ It must be stressed, however, that this result is not strictly analogous to Squire's theorem [15] for incompressible fluids. The correspondence is purely formal since the two-dimensional Dunn-Lin equations do not represent a "proper" two-dimensional disturbance. 
where $r(y), \pi(y), \theta(y), f(y)$, and $\phi(y)$ are the amplitude functions for the perturbations in density, pressure, temperature, and velocities in the $x$ and $y$ directions, respectively; the quantities $U(y), \rho(y), T(y)$, and $\mu(y)$ are the distributions of velocity, density, temperature, and viscosity in the basic flow and can be regarded as known. The ratio of specific heats and the Prandtl number are denoted by $\tau$ and $\sigma$ respectively. Once the solution to the equivalent two-dimensional problem has been obtained, we can recover the solution to the three-dimensional problem by replacing $\alpha$ by $\tilde{\alpha}=\left(\alpha^{2}+\beta^{2}\right)^{1 / 2}$, the Reynolds number $R$ by $\tilde{R}$, and the Mach number $M$ by $\tilde{M}$, where

$$
\tilde{R}=\left(U_{1}{ }^{*} \delta / \nu_{1}{ }^{*}\right) \cos \psi \text { and } \tilde{M}=U_{1}{ }^{*}\left(\tau_{1} R^{*} T_{1}{ }^{*}\right)^{-1 / 2} \cos \psi
$$

In these equations, $\delta$ is the local boundary layer thickness, $R^{*}$ is the gas constant, and $U_{1}{ }^{*}, \nu_{1}{ }^{*}, T_{1}{ }^{*}, \tau_{1}$ are the local free-stream values of the basic velocity, kinematic viscosity, temperature, and the ratio of specific heats, respectively.

Six boundary conditions must be specified in conjunction with Eqs. (2.2) and they are given by

$$
\begin{gathered}
\phi, f, \theta \quad \text { bounded as } y \rightarrow \infty \\
\phi(0)=f(0)=0, \quad a \theta^{\prime}(0)+b \theta(0)=0,
\end{gathered}
$$

where the constants $a$ and $b$ are determined by the thermal properties of the boundary. In particular, the special cases $a=0$ and $b=0$ correspond to the cases of isothermal and insulated walls, respectively.

To facilitate the analysis of Eqs. (2.2), we shall first introduce a Langer variable

$$
\eta(y)=\left\{\frac{3}{2} \int_{\nu_{c}}^{\nu}\left(\frac{U-c}{U_{c}^{\prime}} \frac{\nu_{c}}{\nu}\right)^{1 / 2} d y\right\}^{2 / 3},
$$

where, as usual, we shall suppose that the basic velocity profile $U$ is monotone increasing and $U_{c}^{\prime}=U^{\prime}\left(y_{c}\right) \neq 0, y_{c}$ being a simple zero of $U-c$. A transformation such as (2.5) brings into coincidence the Stokes and anti-Stokes lines associated with the inner and outer expansions and simplifies the subsequent application of certain matching conditions. It should also be noted that this Langer variable is identical to the one used by Dunn [1] except that it has been normalized so that in a neighborhood of $y=y_{c}$, we have

$$
\eta(y)=y-y_{c}+\frac{1}{5}\left(\frac{1}{2} \frac{U_{c}^{\prime \prime}}{U_{c}^{\prime}}-\frac{\nu_{c}{ }^{\prime}}{\nu_{c}}\right)\left(y-y_{c}\right)^{2}+\cdots .
$$

Furthermore, we shall make a preliminary transformation of the dependent variables of the following form:

$$
\begin{gathered}
\phi(y)=\Phi(\eta), \quad f(y)=F(\eta), \quad \theta(y)=\theta(\eta), \\
\pi(y)=\Pi(\eta) \quad \text { and } \rho(y)=R(\eta) .
\end{gathered}
$$

If we let

$$
D=d / d \eta \text { and } \mathscr{L}=\epsilon^{3}\left(D^{2}+\gamma D\right)-\eta,
$$

then, on setting $\sigma=1$, Eqs. (2.2) become

$$
\begin{array}{cl}
\mathscr{L} F=-i g_{1} \Phi+g_{2} \Pi, \quad \mathcal{L} \Phi=g_{3} D \Pi, & \mathcal{L} \theta=-g_{4} \Phi-g_{5} \eta \Pi, \\
g_{6} \eta R+\rho^{\prime} \Phi+\rho\left(i F+\eta^{\prime} D \Phi\right)=0, & \Pi=R / \rho+\theta / T,
\end{array}
$$


where

$$
\begin{gathered}
\gamma(\eta)=\eta^{\prime \prime} / \eta^{\prime 2}, \\
g_{1}(\eta)=\frac{\nu_{c}}{U_{c}{ }^{\prime}} \cdot \frac{U^{\prime}}{\nu \eta^{\prime 2}}, \quad g_{2}(\eta)=\frac{\nu_{c}}{\tau M^{2} U_{c}^{\prime}} \cdot \frac{U^{\prime}}{\mu \eta^{\prime 2}}, \quad g_{3}(\eta)=\frac{\nu_{c}}{i \alpha^{2} \tau M^{2} U_{c}^{\prime}} \cdot \frac{1}{\mu \eta^{\prime}}, \\
g_{4}(\eta)=i \frac{\nu_{c}}{U_{c}{ }^{\prime}} \cdot \frac{T^{\prime}}{\nu \eta^{\prime 2}}, \quad g_{5}(\eta)=\frac{\tau-1}{\tau} \cdot \frac{1}{\rho}, \quad g_{6}(\eta)=i \frac{U_{c}^{\prime}}{\nu_{c}} \nu \eta^{\prime 2} \\
\epsilon=\left(i \alpha R U_{c}^{\prime} / \nu_{c}\right)^{-1 / 3} .
\end{gathered}
$$

Hence, corresponding to the case of neutral stability for which $c$ is real, we have $p h \epsilon=$ $-\pi / 6$. In Eqs. (2.9) and (2.10), primes denote differentiation with respect to $y$. The boundary conditions become

$$
\begin{gathered}
\Phi(\eta), F(\eta), \quad \text { and } \quad \theta(\eta) \text { bounded as } \eta \rightarrow \infty, \\
\Phi\left(\eta_{0}\right)=F\left(\eta_{0}\right)=0, \quad a \eta^{\prime}(0) D \theta\left(\eta_{0}\right)+b \theta\left(\eta_{0}\right)=0,
\end{gathered}
$$

where $\eta_{0}=\eta(0)$. Eqs. (2.9) will then be the starting point of the present analysis

3. The general structure of the uniform approximations. In discussing the solutions of the Dunn-Lin equations, it has been customary to consider, for each of the five disturbance amplitude functions, six linearly independent solutions, two of which are of the inviscid type and four are of the viscous type. In the present work, however, we wish to take into account explicitly certain basic symmetries of the Dunn-Lin equations suggested by a preliminary study of their inner expansions. Specifically, it can be shown that these inner expansions can be represented to all orders in terms of the generalized Airy functions. ${ }^{2}$ This leads us to consider ten exact solutions of Eqs. (2.9) which exhibit precisely the same symmetries in their asymptotic properties as the generalized Airy functions. This also suggests that the uniform approximations to these solutions can be derived asymptotically in terms of these special functions.

In order to simplify the notation in the subsequent discussion, we shall treat the problem of deriving uniform approximations in terms of $\theta(\eta)$ alone. Once the approximations to $\Theta(\eta)$ have been obtained, the corresponding approximations to $\Phi(\eta), F(\eta)$, $R(\eta)$ and $\Pi(\eta)$ can easily be derived. The choice of $\Theta(\eta)$ is to some extent arbitrary since, in principle, the present theory could be carried out in terms of any of the five disturbance functions. Nevertheless, from a study of the structure of the uniform expansions, $\theta(\eta)$ seems to emerge as the most natural variable.

Consider then the ten solutions corresponding to $\theta(\eta)$ which will be denoted by $\theta_{W}(\eta), \theta_{B, k}(\eta), \theta_{M, k}(\eta)$ and $\theta_{T, k}(\eta)(k=1,2,3)$. We now require that $\theta_{W}(\eta)$ be wellbalanced, $\Theta_{B, k}(\eta)$ be balanced in $T_{k}$, and that both $\Theta_{M, k}$ and $\theta_{T, k}$ be recessive in $S_{k}$, where $T_{k}$ and $S_{k}$ are sectors in the complex plane bounded by Stokes and anti-Stokes lines respectively (see Fig. 1). The asymptotic properties of $\theta_{W}(\eta)$ and $\theta_{B, k}(\eta)$ then uniquely define these solutions to within a multiplicative factor and modulo a multiple of $\theta_{W}(\eta)$ in the case of $\theta_{B, k}(\eta)$. The solutions $\theta_{M, k}(\eta)$ and $\theta_{r, k}(\eta)$ are, however, indistinguishable by their asymptotic properties alone. Additional conditions are needed for their unique characterization, and these will be given in the form of certain matching conditions in conjunction with our discussion of their outer expansions in Sec. 4. The

${ }^{2}$ For a complete discussion of the definitions and properties of the generalized Airy functions, see [11]. 




Fig. 1. The anti-Stokes lines (left) and the Stokes lines (right) in the $\eta$-plane.

presence of two solutions of dominant-recessive type in any one sector $S_{k}$ is perhaps a distinctive feature of the Dunn-Lin equations as well as other two-dimensional stability equations of compressible boundary layer theory. This fact also leads to certain important differences between the asymptotic theory of the Dunn-Lin equations and that of the Orr-Sommerfeld equation.

The general structure of the uniform approximations to these solutions can now be inferred directly from their corresponding inner expansions. For our purpose the explicit forms of these inner expansions will not be needed. We need only note that, to the first three orders, the inner expansions of $\theta_{B, k}(\eta)$ can be expressed in terms of the generalized Airy functions $B_{k}(\zeta ; p, 1)$ (where $\zeta=\eta / \epsilon$ ) for certain integral values of $p$ and, by using the recursion formula satisfied by these functions, it is then sufficient to let $p$ take on the values $1,0,-1$ (say). The structure of the inner expansions then suggests the uniform approximations of balanced type must be of the form

$$
\begin{aligned}
\Theta_{B, k}(\eta)=\mathcal{G}(\eta, \epsilon)-\left\{Q(\eta, \epsilon) B_{k}(\zeta ; 1,1)+\epsilon^{-1} \mathbb{B}(\eta, \epsilon) B_{k}(\zeta ; 0,1)\right. \\
\left.\quad+\epsilon \mathcal{C}(\eta, \epsilon) B_{k}(\zeta ;-1,1)\right\}+0\left(\epsilon^{2}\right),
\end{aligned}
$$

where $Q, B, \mathcal{C}$, and $\mathcal{G}$ have asymptotic power series expansions of the form

$$
a(\eta, \epsilon)=\sum_{k=0}^{\infty} \epsilon^{3 k} a_{k}(\eta)
$$

and where the coefficients $Q_{k}(\eta)$ are analytic at $\eta=0 .^{3}$ For convenience, we shall now omit the subscripts on $a_{0}(\eta), B_{0}(\eta), \mathfrak{C}_{0}(\eta)$, and $\mathcal{S}_{0}(\eta)$, and in a "first approximation" to $\theta_{B, k}(\eta)$, we have

$$
\theta_{B, k}(\eta)=S(\eta)-\left\{Q(\eta) B_{k}(\zeta ; 1,1)+\epsilon^{-1} B(\eta) B_{k}(\zeta ; 0,1)\right\} .
$$

The corresponding first approximation to the derivative of $\theta_{B, k}(\eta)$ is given by

${ }^{3}$ To be more precise, the coefficient $S(\eta, \epsilon)$ must be expanded in terms of the asymptotic sequence $\left\{\log \epsilon, 1, \epsilon^{3} \log ^{2} \epsilon, \epsilon^{3} \log \epsilon, \epsilon^{3}, \cdots\right\}$. Hence $\varphi(\eta, \epsilon)=\varphi_{0 l}(\eta) \log \epsilon+\varphi_{01}(\eta)+0\left(\epsilon^{3} \log ^{2}, \epsilon^{3} \log \epsilon, \epsilon^{3}\right)$ (say). For the present purpose, however, it is not necessary to make such an explicit decomposition (cf. Eq. $(5.20))$. 


$$
\begin{aligned}
\Theta_{B, k}{ }^{\prime}(\eta)= & \mathcal{S}^{\prime}(\eta)-\mathcal{e}(\eta)-\left\{Q^{\prime}(\eta) B_{k}(\zeta ; 1,1)\right. \\
& \left.+\epsilon^{-1}\left[\mathfrak{Q}(\eta)+B^{\prime}(\eta)+\eta \mathfrak{C}(\eta)\right] B_{k}(\zeta ; 0,1)+\epsilon^{-2} \leftrightarrow(\eta) B_{k}(\zeta ;-1,1)\right\} .
\end{aligned}
$$

Hence the problem of obtaining uniform approximations to the solutions of balanced type and their first derivatives can be reduced to the determination of the four coefficients $Q(\eta), B(\eta), \mathcal{e}(\eta)$, and $G(\eta)$.

The structure of the expansions of dominant-recessive type can be inferred either from the inner expansions of $\theta_{M, k}(\eta)$ and $\theta_{T, k}(\eta)$, or directly from Eq. (3.1) by using the connection formula satisfied by the generalized Airy functions (cf. [11]). Thus, corresponding to Eq. (3.1), we have

$$
\begin{gathered}
\Theta_{M, k}(\eta)=a_{M}(\eta) A_{k}(\zeta ; 1)+\epsilon^{-1} b_{M}(\eta) A_{k}(\zeta ; 0)+\epsilon c_{M}(\eta) A_{k}(\zeta ;-1), \\
\Theta_{T, k}(\eta)=a_{T}(\eta) A_{k}(\zeta ; 1)+\epsilon^{-1} b_{T}(\eta) A_{k}(\zeta ; 0)+\epsilon c_{T}(\eta) A_{k}(\zeta ;-1),
\end{gathered}
$$

where the coefficients are the leading terms of expansions of the form given by (3.2). In a first approximation, terms involving $c_{M}(\eta)$ and $c_{T}(\eta)$ must, of course, be omitted from Eqs. (3.5) and (3.6), respectively. They are needed, however, in the first approximations to $\theta_{M, k}{ }^{\prime}(\eta)$ and $\theta_{T, k}{ }^{\prime}(\eta)$. It is perhaps of some interest to note that with our subsequent normalization of $\Theta_{M, k}(\eta)$ and $\theta_{T, k}(\eta)$, the leading terms in their inner expansions will correspond, respectively, to certain viscous approximations of the momentum and thermal groups in the older heuristic theories (cf. [14]). This also explains our choice of subscripts for $\theta_{M}(\eta)$ and $\theta_{T}(\eta)$.

The structures of the uniform approximations for the other dependent variables are essentially identical to those of $\theta(\eta)$ except that in the cases of $\Phi(\eta)$ and $\Pi(\eta)$, the factor $\epsilon^{-1}$ in terms involving $A_{k}(\zeta ; 0)$ and $B_{k}(\zeta ; 0,1)$ must be replaced by $\epsilon^{2}$.

It would have been possible, of course, to express the expansions of balanced and dominant-recessive types respectively in terms of $B_{k}(\zeta ; p, 1)$ and $A_{k}(\zeta ; p)$ with $p=2$, 1,0 , say, rather than $1,0,-1$. Our present choice of $p=1,0,-1$, however, seems to be the most convenient one for $\Theta(\eta)$ partly because of their compatibility with certain outer expansions to be defined in Sec. 4, and partly because they lead directly to a simple derivation of the coefficients $Q(\eta), a_{M}(\eta)$, and $a_{T}(\eta)$. For these reasons, $\Theta(\eta)$ also appears to be the most natural dependent variable associated with the problem.

The ten solutions which we have defined for $\Theta(\eta)$ cannot, of course, be linearly independent, but must satisfy four exact connection formulae. Although these connection formulae are not essential to the development of the present theory, as has been shown by Reid $[12,13]$ in his discussion of the Orr-Sommerfeld equation, nevertheless their use can lead to a more systematic derivation of the coefficients in the expansions of balanced type. Thus, for example, by using the connection formula

$$
B_{2}(\zeta ; p, 1)-B_{3}(\zeta ; p, 1)=2 \pi i A_{1}(\zeta ; p),
$$

we obtain to first order

$$
\theta_{B, 2}(\eta)-\theta_{B, 3}(\eta)=s_{M} \theta_{M, 1}(\eta)+s_{T} \theta_{T, 1}(\eta),
$$

where $s_{M}$ and $s_{T}$ are two appropriately chosen constants which depend on the normalizations of $\theta_{B, k}(\eta), \theta_{M, k}(\eta)$, and $\theta_{T, k}(\eta)$. On substituting the expansions (3.1), (3.5), and (3.6) into Eq. (3.8), it then follows that the coefficients in the expansions of balanced and dominant-recessive types must satisfy relations of the form

$$
Q(\eta)=s_{M} a_{M}(\eta)+s_{T} a_{T}(\eta) .
$$


Hence the coefficients in the expansions of balanced type can be derived from the corresponding coefficients in the expansions of dominant-recessive type.

4. The outer expansions. In order to achieve further simplifications in the derivation of the slowly varying coefficients in (3.1), (3.5), and (3.6), it will be necessary to match these approximations in suitably restricted domains of the $\eta$-plane to their corresponding outer expansions. For this purpose, we need to construct ten outer expansions for $\theta(\eta)$ which correspond, in their asymptotic properties, to the ten solutions defined in Sec. 3. The methods of deriving these outer expansions are essentially the same as those used in the older heuristic theories to obtain approximations of inviscid and viscous types which are valid away from the turning point. But the results presented here are new in so far as they have been derived for the case where the Prandtl number is one. The role these expansions play in the present theory is also substantially different from that in the older theories.

The outer expansions of well-balanced and balanced types. We may obtain outer expansions of well-balanced and balanced types by first considering, for each dependent variable $\bar{Q}$ (say), an expansion of the form

$$
\bar{Q}(\eta, \epsilon)=\sum_{k=0}^{\infty} \epsilon^{3 k} \bar{Q}^{(3 k)}(\eta) .
$$

On substituting (4.1) into Eqs. (2.9), we find that to the lowest order $\bar{Q}^{(0)}(\eta)$ satisfies a system of equations which is identical to the so-called inviscid equations obtained from (2.9) by letting $|\epsilon| \rightarrow 0$. These equations can be further uncoupled and rewritten as a single second-order equation in terms of $\Phi^{(0)}(\eta)$ or $\Pi^{(0)}(\eta)$. In terms of $\Phi^{(0)}(\eta)$, we have

$$
\left[\eta D^{2}+p(\eta) D+q(\eta)\right] \Phi^{(0)}(\eta)=0,
$$

where $p(\eta)$ and $q(\eta)$ have Taylor series expansions of the form

$$
p(\eta)=\sum_{k=1}^{\infty} p_{k} \eta^{k}, \quad q(\eta)=\sum_{k=0}^{\infty} q_{k} \eta^{k} .
$$

Two solutions of Eq. (4.2) can be obtained by the method of Frobenius and they can be written as

$$
\begin{gathered}
\Phi_{W}{ }^{(0)}(\eta)=\eta \Phi_{W}(\eta), \\
\Phi_{B}{ }^{(0)}(\eta)=\bar{\phi}_{B}(\eta)+\left(5 \gamma_{0}-\frac{T_{c}{ }^{\prime}}{T_{c}}+2 \frac{\nu_{c}{ }^{\prime}}{\nu_{c}}\right) \Phi_{W}{ }^{(0)}(\eta) \log \eta,
\end{gathered}
$$

where $\bar{\phi}_{W}(\eta)$ and $\bar{\phi}_{B}(\eta)$ are power series in $\eta$ with the leading terms normalized to unity for convenience. Moreover, $\Phi_{L}{ }^{(0)}(\eta)$ is made definite by requiring that $\Phi_{B}(\eta)$ contains no multiple of $\Phi_{W}{ }^{(0)}(\eta)$, i.e. the coefficient of the linear term in $\Phi_{B}(\eta)$ is zero. A simple calculation then gives

$$
\begin{gathered}
\bar{\Theta}_{W}{ }^{(0)}(\eta)=i \frac{T_{c}{ }^{\prime}}{U_{c}{ }^{\prime}}\left\{1+\frac{T_{c}{ }^{\prime \prime}}{T_{c}{ }^{\prime}} \eta+0\left(\eta^{2}\right)\right\}, \\
\bar{\Theta}_{B}{ }^{(0)}(\eta)=\bar{\theta}_{B}(\eta)+\left(5 \gamma_{0}-\frac{T_{c}{ }^{\prime}}{T_{c}}+2 \frac{\nu_{c}{ }^{\prime}}{\nu_{c}}\right) \bar{\Theta}_{W}{ }^{(0)}(\eta) \log \eta,
\end{gathered}
$$


where

$$
\bar{\theta}_{B}(\eta)=i \frac{T_{c}{ }^{\prime}}{U_{c}{ }^{\prime}}\left\{\frac{1}{\eta}+0(1)\right\} .
$$

The normalizations of $\bar{\Theta}_{W}^{(0)}(\eta)$ and $\bar{\theta}_{B}{ }^{(0)}(\eta)$ are completely fixed in terms of those of $\Phi_{W}{ }^{(0)}$ and $\Phi_{B}{ }^{(0)}(\eta)$.

Since $\bar{\theta}_{W}^{(0)}(\eta)$ is regular at $\eta=0$, it is clear that it provides a uniform approximation to the well-balanced solution $\theta_{W}(\eta)$ defined in Sec. 3. But on the other hand, $\bar{\theta}_{B}{ }^{(\dot{ })}(\eta)$ has a logorithmic branch point at $\eta=0$, and hence it cannot be uniformly valid in a full neighborhood of the turning point at which the Dunn-Lin equations are regular. Following the suggestion of Reid [11], we may, however, restrict $p h \eta$ such that $-3 \pi / 2<$ $p h \eta<\pi / 2$ and define three outer expansions $\bar{\theta}_{B, k}(\eta)(k=1,2,3)$ according to the following scheme:

$$
\bar{\Theta}_{B, k}(\eta)=\bar{\theta}_{B}{ }^{(0)}(\eta) \quad\left(\eta \in T_{k}\right) .
$$

Thus the $\bar{\theta}_{B, k}(\eta)$ are balanced in $T_{k}$ and provide the required outer expansions.

The outer expansions of dominant-recessive type. In order to derive the outer expansions of dominant-recessive type, it has been customary to use expansions in inverse power of $\lambda=\epsilon^{-3 / 2}$. By a preliminary calculation which is essentially a direct application of the well-known WKB method, we find that $\bar{\theta}(\eta)$ must be of the form

$$
\bar{\Theta}^{ \pm}(\eta, \epsilon)=C \exp \left( \pm \frac{2}{3} \lambda \eta^{3 / 2}\right) \sum_{k=0}^{\infty}( \pm \lambda)^{-k} \bar{\Theta}^{(k)}(\eta)
$$

where $C$ is an overall normalization constant. On substituting (4.10) and similar expansions for the other dependent variables into Eqs. (2.9), we obtain a sequence of systems of differential equations from which the Poincaré part of these expansions can be determined to all orders. The lowest order system of equations is homogeneous and can be written as a single second-order differential equation in terms of $\bar{\theta}^{(0)}(\eta)$ of the form

$$
\mathfrak{N} \bar{\Theta}^{(0)}=0, \quad \Re=\eta D^{2}+r(\eta) D+\frac{1}{\eta} s(\eta)
$$

and $r(\eta)$ and $s(\eta)$ have Taylor series expansions of the form

$$
r(\eta)=2+\sum_{k=1}^{\infty} r_{k} \eta^{k}, \quad s(\eta)=\frac{3}{16}+\sum_{k=1}^{\infty} s_{k} \eta^{n}
$$

It is interesting to note here that the structure of these outer expansions is quite similar to those encountered in the study of the stability of spiral flow between rotating cylinders (cf. [3]) and in both cases their structure depends crucially on the roots of the indicial equation associated with a certain second-order equation which is singular at $\eta=0$. For Eq. (4.11), the exponents are $-3 / 4$ and $-1 / 4$, and hence we obtain two solutions of the form

$$
\bar{\theta}_{M}{ }^{(0)}(\eta)=\eta^{-3 / 4} \bar{\theta}_{M}(\eta) \text { and } \bar{\theta}_{T}^{(0)}(\eta)=\eta^{-1 / 4} \bar{\theta}_{T}(\eta),
$$

where $\bar{\theta}_{M}(\eta)$ and $\bar{\theta}_{T}(\eta)$ are power series in $\eta$ with the leading terms normalized to $i T_{c}{ }^{\prime} / U_{c}{ }^{\prime}$ for convenience, i.e.

$$
\bar{\theta}_{M}(\eta)=i \frac{T_{c}{ }^{\prime}}{U_{c}^{\prime}}\left\{1+\alpha_{M} \eta+0\left(\eta^{2}\right)\right\}, \quad \bar{\theta}_{T}(\eta)=i \frac{T_{c}^{\prime}}{U_{c}^{\prime}}\left\{1+\alpha_{T} \eta+0\left(\eta^{2}\right)\right\} .
$$


The constants $\alpha_{M}$ and $\alpha_{T}$ are given by

$$
\alpha_{M}=\frac{1}{2}\left(7 \gamma_{0}+\frac{T_{c}{ }^{\prime}}{T_{c}}+2 \frac{\nu_{c}{ }^{\prime}}{\nu_{c}}-2 \frac{T_{c}{ }^{\prime \prime}}{T_{c}{ }^{\prime}}\right), \quad \alpha_{T}=-\frac{1}{2}\left(\gamma_{0}-\frac{2}{3} \cdot \frac{T_{c}{ }^{\prime}}{T_{c}}\right) .
$$

The system of equations for the second-order terms in these outer expansions is not homogeneous but it can also be uncoupled and rewritten as a second-order equation involving only $\bar{\Theta}^{(1)}(\eta)$ and $\bar{\Theta}^{(0)}(\eta)$ of the form

$$
\mathfrak{M} \bar{\Theta}^{(1)}=\mathscr{g}\left(\bar{\Theta}^{(0)}\right) \text {. }
$$

If we let

$$
\mathfrak{J}_{M}=\mathfrak{g}\left(\bar{\theta}_{M}{ }^{(0)}\right), \quad \mathfrak{g}_{T}=\mathfrak{g}\left(\bar{\theta}_{T}{ }^{(0)}\right)
$$

then we can derive two particular solutions $\bar{\Theta}_{M}{ }^{(1)}$ and $\bar{\Theta}_{T}{ }^{(1)}$ of Eq. (4.16), corresponding to $\bar{\theta}_{M}{ }^{(0)}(\eta)$ and $\bar{\Theta}_{T}{ }^{(0)}(\eta)$. The exact forms of the inhomogeneous terms $\mathscr{I}_{M}$ and $\mathscr{g}_{T}$ are known, but they are somewhat lengthy and hence will not be given here. Moreover, $\bar{\Theta}_{M}{ }^{(1)}(\eta)$ will not be needed for the purpose of matching and it suffices therefore to note that

$$
\jmath_{T}=i \frac{5}{32} \cdot \frac{T_{c}{ }^{\prime}}{U_{c}{ }^{\prime}} \eta^{-7 / 4}\left\{1-\left(\frac{9}{2} \gamma_{0}-\frac{1}{6} \cdot \frac{T_{c}{ }^{\prime}}{T_{c}}+\frac{3}{2} \cdot \frac{\nu_{c}{ }^{\prime}}{\nu_{c}}-\frac{T_{c}{ }^{\prime \prime}}{T_{c}{ }^{\prime}}\right) \eta+0\left(\eta^{2}\right)\right\} .
$$

By using the method of variation of parameters, it is found that

$$
\begin{aligned}
\bar{\theta}_{T}{ }^{(1)}(\eta)=i \frac{T_{c}{ }^{\prime}}{U_{c}{ }^{\prime}} \eta^{-7 / 4}\left\{\frac{5}{48}+\left(\frac{5}{12} \alpha_{T}-\frac{5}{16} \alpha_{M}\right) \eta\right. & +\cdots\} \\
& +h_{M} \bar{\theta}_{M}{ }^{(0)}(\eta)+h_{T} \bar{\theta}_{T}{ }^{(0)}(\eta),
\end{aligned}
$$

where $h_{M}$ and $h_{T}$ are arbitrary constants which can be fixed in various ways to be discussed in the following section. form

Combining these results, we then have four WKB approximations for $\bar{\theta}(\eta)$ of the

$$
\begin{gathered}
\bar{\Theta}_{M}{ }^{ \pm}(\eta)=\frac{1}{2} \pi^{-1 / 2} \epsilon^{3 / 4} \exp \left( \pm \frac{2}{3} \lambda \eta^{3 / 2}\right) \sum_{\substack{k=0 \\
\infty}}^{\infty}( \pm \lambda)^{k} \bar{\Theta}_{M}{ }^{(k)}(\eta) \\
\bar{\Theta}_{T}{ }^{ \pm}(\eta)=\frac{1}{2} \pi^{-1 / 2} \epsilon^{-3 / 4} \exp \left( \pm \frac{2}{3} \lambda \eta^{3 / 2}\right) \sum_{k=0}^{\infty}( \pm \lambda)^{k} \bar{\Theta}_{T}{ }^{(k)}(\eta)
\end{gathered}
$$

where the overall normalizations have been fixed in order to facilitate the subsequent matching. We can now define six outer expansions, $\bar{\theta}_{M, k}(\eta)$ and $\bar{\theta}_{T, k}(\eta)(k=1,2,3)$, in terms of $\bar{\theta}_{M}{ }^{ \pm}(\eta)$ and $\bar{\theta}_{T}{ }^{ \pm}(\eta)$, corresponding to the six solutions $\theta_{M, k}(\eta)$ and $\theta_{T, k}(\eta)$ $(k=1,2,3)$. For this purpose, we shall regard $\bar{\theta}_{M}{ }^{ \pm}(\eta)$ and $\bar{\theta}_{T}{ }^{ \pm}(\eta)$ to be defined for all values of $\eta$ in the range $-3 \pi / 2<p h \eta<\pi / 2$. The outer expansions $\bar{\theta}_{M, k}(\eta)(k=1,2,3)$ which are recessive in $S_{k}$ can then be defined by the following scheme:

$$
\begin{aligned}
\bar{\Theta}_{M, 1}(\eta) & =\bar{\theta}_{M}{ }^{-}(\eta) & & \left(\eta \in T_{2} \cup T_{3}\right), \\
\bar{\Theta}_{M, 2}(\eta) & =i \bar{\theta}_{M}{ }^{+}(\eta) & & \left(\eta \in T_{3} \cup T_{1}\right), \\
\bar{\Theta}_{M, 3}(\eta) & =-i \bar{\Theta}_{M}{ }^{+}(\eta) & & \left(\eta \in T_{2}\right), \\
& =-\bar{\theta}_{M}{ }^{-}(\eta) & & \left(\eta \in T_{1}\right) .
\end{aligned}
$$


The remaining three outer expansions $\bar{\theta}_{T, k}(\eta)(k=1,2,3)$ can be defined in a similar manner in terms of $\bar{\theta}_{T}{ }^{ \pm}(\eta)$. Moreover, the dominant-recessive type solutions $\theta_{M, k}(\eta)$ and $\Theta_{T, k}(\eta)$ can now be uniquely defined if we require that their outer expansions be given by $\bar{\theta}_{M, k}$ and $\bar{\theta}_{T, k}(\eta)$, respectively.

5. Determination of the coefficients in the uniform approximations. In order to derive the slowly varying coefficients in the uniform approximations, we may consider, of course, the direct method of substituting these approximations into the Dunn-Lin equations. Depending on the type of approximations involved, we would then obtain three or four sets of coupled differential equations for the coefficients, all but one of which are of rather complicated form. Alternatively, we observe that these approximations, being uniform, are expected to be reducible to their corresponding outer expansions within certain common domains of validity. This then suggests that the procedure for determining the required coefficients can be partially simplified by matching the uniform approximations to their outer expansions defined in Sec. 4. It should be stressed, however, that matching can yield some but not all of the coefficients needed. Differential equations satisfied by the coefficients must be used to derive $\alpha(\eta), a_{M}(\eta)$ and $a_{T}(\eta)$ in (3.1), (3.5), and (3.6). Nevertheless, this approach has the distinct advantage of enabling us to normalize the uniform approximations in a natural manner. It also brings out explicitly the relations between the results of the present and the older theories. In view of the relation (3.9), it is perhaps more convenient to consider first the approximations of dominant-recessive type.

The dominant-recessive approximations of $M$-type. On substituting (3.5) and the corresponding expansions of the other dependent variables into Eqs. (2.9), we obtain a system of homogeneous differential equations for the coefficients, among which the equations involving the coefficients of $A_{k}(\zeta ; 1)$ form a closed system that can immediately be identified as the "inviscid equations" discussed in Sec. 4 . Hence we must have

$$
a_{M}(\eta)=a_{M}(0) \bar{\Theta}_{W}{ }^{(0)}(\eta)
$$

where $\bar{\theta}_{W}{ }^{(0)}(\eta)$ is simply the well-known regular inviscid solution of the Dunn-Lin equations given by (4.6).

We may, of course, derive $b_{M}(\eta)$ and $c_{M}(\eta)$ in a similar way. For example, it is found that $b_{M}(\eta)$ must be a multiple of the regular solution of the following homogeneous equation:

$$
\left\{\eta D^{2}+u(\eta) D+v(\eta)\right\} \Psi=0,
$$

where

$$
\begin{aligned}
u(\eta)=\frac{3}{2}+\eta\left(5 \gamma-\frac{1}{2} \cdot \frac{T^{\prime}}{T \eta^{\prime}}+\frac{3}{2} \cdot \frac{\nu^{\prime}}{\nu \eta^{\prime}}-\frac{T^{\prime \prime}}{T^{\prime} \eta^{\prime}}\right), & \\
v(\eta)=\frac{1}{2}\left(\frac{3}{2} \gamma-\frac{T^{\prime}}{T \eta^{\prime}}\right)+\frac{\eta}{2} & {\left[\frac{\eta^{\prime \prime \prime}}{\eta^{\prime 2}}+\frac{5}{2} \gamma^{2}\right.} \\
& \left.\quad-\gamma\left(\frac{5}{2} \cdot \frac{T^{\prime}}{T \eta^{\prime}}-\frac{3}{2} \cdot \frac{\nu^{\prime}}{\nu \eta^{\prime}}+\frac{T^{\prime \prime}}{T^{\prime} \eta^{\prime}}\right)+\frac{T^{\prime}}{T \eta^{\prime}}\left(\frac{T^{\prime}}{T \eta^{\prime}}-\frac{\nu^{\prime}}{\nu \eta^{\prime}}\right)\right]
\end{aligned}
$$

while $c_{M}(\eta)$ must satisfy a somewhat lengthy inhomogeneous equation in which the inhomogeneous term involves $a_{M}(\eta)$ and $b_{M}(\eta)$. Thus, in principle, the coefficients 
$a_{M}(\eta), b_{M}(\eta)$, and $c_{M}(\eta)$ can be completely determined (up to a normalization factor in the cases of $a_{M}(\eta)$ and $\left.b_{M}(\eta)\right)$ by certain differential equations. The necessity of solving the latter two equations can, however, be avoided if we now match approximations of the form (3.5) to the corresponding outer expansions in their respective domains of validity. Hence a re-expansion of $\theta_{M, 1}(\eta)$ for $\eta$ in $\left(I-T_{1}\right) \cap(|\epsilon| \prec|\eta| \precsim 1)$ gives

$$
\begin{aligned}
\Theta_{M, 1}(\eta) & \sim \frac{1}{2} \pi^{-1 / 2} \epsilon^{-3 / 4} \exp \left(-\frac{2}{3} \epsilon^{-3 / 2} \eta^{3 / 2}\right) \\
& \times\left\{\eta^{-1 / 4} b_{M}(\eta)-\epsilon^{3 / 2}\left[\frac{5}{48} \eta^{-7 / 4} b_{M}(\eta)+\eta^{-3 / 4} a_{M}(\eta)+\eta^{1 / 4} c_{M}(\eta)\right]+0\left(\epsilon^{3}\right)\right\} .
\end{aligned}
$$

Combining the results of (4.20) and (4.22), we obtain an outer expansion of the form

$$
\frac{1}{2} \pi^{-1 / 2} \epsilon^{3 / 4} \exp \left(-\frac{2}{3} \epsilon^{-3 / 2} \eta^{3 / 2}\right)\left\{\bar{\Theta}_{M}{ }^{(0)}(\eta)+0\left(\epsilon^{3 / 2}\right)\right\},
$$

where

$$
\bar{\theta}_{M}{ }^{(0)}(\eta)=\eta^{-3 / 4} \bar{\theta}_{M}(\eta) .
$$

If we now require that (5.3) and (5.4) be identical, thereby fixing the normalization of $\theta_{M, 1}(\eta)$ in terms of its outer expansion, and require that $a_{M}(\eta), b_{M}(\eta)$, and $c_{M}(\eta)$ be analytic at $\eta=0$, then we must have

$$
a_{M}(0)=-1, \quad b_{M}(\eta) \equiv 0, \quad c_{M}(\eta)=-\eta^{-1}\left\{\theta_{M}(\eta)+a_{M}(\eta)\right\} .
$$

These results, incidentally, also clearly show that the use of differential equations at some point of the present theory is essential. Otherwise the coefficient $c_{M}(\eta)$ cannot be determined by matching alone.

The dominant-recessive approximations of T-type. The coefficients in the uniform expansions of $\Theta_{r, k}(\eta)$ can now be determined in a similar manner. Clearly, $a_{T}(\eta), b_{T}(\eta)$, and $c_{T}(\eta)$ satisfy the same equations as $a_{M}(\eta), b_{M}(\eta)$, and $c_{M}(\eta)$. We shall, however, make no essential use of this fact for reasons we have already discussed, except to note that $a_{T}(\eta)$ is given by

$$
a_{T}(\eta)=a_{T}(0) \bar{\Theta}_{W}{ }^{(0)}(\eta),
$$

where $a_{T}(0)$ is the normalization constant to be determined later. Again, by re-expanding $\Theta_{T, 1}(\eta)$ for values of $\eta$ in $\left(I-T_{1}\right) \cap(|\epsilon| \prec|\eta| \precsim 1)$, we obtain

$$
\begin{aligned}
\Theta_{T, 1}(\eta) & \sim \frac{1}{2} \pi^{-1 / 2} \epsilon^{-3 / 4} \exp \left(-\frac{2}{3} \epsilon^{-3 / 2} \eta^{3 / 2}\right) \\
& \times\left\{\eta^{-1 / 4} b_{T}(\eta)-\epsilon^{3 / 2}\left[\frac{5}{48} \eta^{-7 / 4} b_{T}(\eta)+\eta^{-3 / 4} a_{T}(\eta)+\eta^{1 / 4} c_{T}(\eta)\right]+0\left(\epsilon^{3}\right)\right\} .
\end{aligned}
$$

On the other hand, the outer expansion of $\Theta_{T, 1}(\eta)$ derived in Sec. 4 is of the form

$$
\frac{1}{2} \pi^{-1 / 2} \epsilon^{-3 / 4} \exp \left(-\frac{2}{3} \epsilon^{-3 / 2} \eta^{3 / 2}\right)\left\{\bar{\theta}_{T}^{(0)}(\eta)-\epsilon^{3 / 2} \bar{\theta}_{T}^{(1)}(\eta)+0\left(\epsilon^{3}\right)\right\},
$$

where

$$
\begin{gathered}
\bar{\theta}_{T}{ }^{(0)}(\eta)=\eta^{-1 / 4} \bar{\theta}_{T}(\eta), \\
\bar{\theta}_{T}{ }^{(1)}(\eta)=i \frac{T_{c}{ }^{\prime}}{U_{c}{ }^{\prime}} \eta^{-7 / 4}\left\{\frac{5}{48}+\left(\frac{5}{12} \alpha_{T}-\frac{5}{16} \alpha_{M}\right)+\cdots\right\}+h_{M} \bar{\theta}_{M}{ }^{(0)}(\eta)+h_{T} \bar{\theta}_{T}{ }^{(0)}(\eta)
\end{gathered}
$$

such that $\alpha_{M}$ and $\alpha_{T}$ are constants given by (4.15). By requiring that (5.8) and (5.9) be 
identical we can immediately deduce that

$$
b_{T}(\eta)=\bar{\theta}_{T}(\eta), \quad c_{T}(\eta)=\eta^{-2}\left\{\eta^{7 / 4} \bar{\theta}_{T}^{(1)}(\eta)-\eta a_{T}(\eta)-\frac{5}{48} b_{T}(\eta)\right\} .
$$

The behavior of $c_{T}(\eta)$ as $\eta \rightarrow 0$ is then given by

$$
c_{T}(\eta)=-i \frac{T_{c}{ }^{\prime}}{U_{c}{ }^{\prime}}\left\{\eta^{-1}\left[\frac{s}{16}\left(\alpha_{M}-\alpha_{T}\right)-h_{M}+a_{T}(0)\right]-\eta^{-1 / 2} h_{T} \theta_{T}(\eta)+0(1)\right\},
$$

and our requirement that $c_{T}(\eta)$ be analytic at $\eta=0$ then shows that the coefficients of $\eta^{-1}$ and $\eta^{-1 / 2}$ must both vanish identically. Hence we have

$$
h_{T}=0, \quad h_{M}-a_{T}(0)=\frac{5}{16}\left(\alpha_{M}-\alpha_{T}\right) .
$$

This regularity condition, however, does not fix $h_{M}$ and $a_{T}(0)$ separately. One way to fix these constants would be to require that, in a first approximation, the solutions $\theta_{T, k}(\eta)$ contain no multiple of $\theta_{M, k}(\eta)$, which then implies that

$$
a_{T}(0)=0, \quad h_{M}=\frac{5}{16}\left(\alpha_{M}-\alpha_{T}\right) .
$$

This particular normalization of $\theta_{T, k}(\eta)$ will serve the purpose of simplifying somewhat the constants $s_{M}$ and $s_{T}$ in equation (3.9). But on the other hand, it might be more desirable for some purposes to fix the normalization of $\theta_{r, k}(\eta)$ by considering the corresponding outer expansions of the form (4.22). In that case, the customary procedure is to require that $\bar{\theta}_{T}{ }^{(1)}(\eta)$ contains no multiple of the solutions of the homogeneous equation (4.11). We must then let $h_{T}=h_{M}=0$ and $a_{T}(0)=-(5 / 16)\left(\alpha_{M}-\alpha_{T}\right)$.

The approximations of well-balanced and balanced types. For the solution of wellbalanced type, it is clear that a first-order uniform approximation to $\theta_{W}(\eta)$ is provided by the first term of its outer expansion. Thus we simply have

$$
\theta_{W}(\eta)=\bar{\theta}_{W}{ }^{(0)}(\eta)+0\left(\epsilon^{3}\right),
$$

where $\bar{\theta}_{W}{ }^{(0)}(\eta)$ is the regular inviscid solution given by Eq. (4.6).

The coefficients $Q(\eta), B(\eta)$, and $\mathfrak{e}(\eta)$ in the approximations of balanced type can, of course, be determined by certain differential equations which are identical to those satisfied by the corresponding coefficients in the approximations of dominant-recessive type. Moreover, it can easily be verified that the well-balanced part $G(\eta)$ must be the solution of a certain inhomogeneous version of the inviscid equations. Alternatively, these coefficients can be derived more systematically by using the relations of the form (3.9). For this purpose, it is necessary to first determine the constants $s_{M}$ and $s_{T}$ in (3.9) by matching. We shall also suppose that the normalization of $\theta_{T, k}(\eta)$ is fixed by Eq. (5.15).

Consider then the uniform expansion of $\theta_{B, 3}(\eta)$ (say) given by Eq. (3.1). On substituting relations of the form (3.9) and re-expanding for $\eta$ in $T_{3} \cap(|\epsilon| \prec|\eta| \preccurlyeq 1)$, we obtain

$\theta_{B, 3}(\eta) \sim\left\{s_{T} \frac{\bar{\theta}_{T}(\eta)}{\eta}+\mathcal{G}(\eta)+s_{M}[\log \epsilon-\gamma+2 \pi i] \bar{\theta}_{W}{ }^{(0)}(\eta)\right\}-s_{M} \bar{\theta}_{W}{ }^{(0)}(\eta) \log \eta$,

where $\gamma$ is Euler's constant. But on the other hand, from (4.7) and (4.9), the outer expansion of $\theta_{B, 3}(\eta)$ is given by 


$$
\bar{\theta}_{B, 3}{ }^{(0)}(\eta)=\bar{\theta}_{B}(\eta)+\left(5 \gamma_{0}-\frac{T_{c}{ }^{\prime}}{T_{c}}+2 \frac{\nu_{c}{ }^{\prime}}{\nu_{c}}\right) \bar{\theta}_{W}{ }^{(0)}(\eta) \log \eta .
$$

Again if we require that Eqs. (5.17) and (5.18) be identical, thereby fixing the normalization of $\theta_{B, 3}(\eta)$ in terms of its outer expansion, we must have

$$
s_{M}=-\left(5 \gamma_{0}-\frac{T_{c}{ }^{\prime}}{T_{c}}+2 \frac{\nu_{c}{ }^{\prime}}{\nu_{c}}\right), \quad s_{T}=1
$$

so that the well-balanced part $\mathcal{G}(\eta)$ of $\theta_{B, 3}(\eta)$ is given by

$$
\mathcal{S}(\eta)=\bar{\theta}_{B}(\eta)-\frac{\bar{\theta}_{T}(\eta)}{\eta}+\left(5 \gamma_{0}-\frac{T_{c}{ }^{\prime}}{T_{c}}+2 \frac{\nu_{c}{ }^{\prime}}{\nu_{c}}\right)[\log \epsilon-\gamma+2 \pi i] \bar{\Theta}_{W}{ }^{(0)}(\eta) .
$$

It can then be easily verified that $g(\eta)$ is regular at $\eta=0$. On substituting Eqs. (5.19) into relations of the form (3.9), we have

$$
\begin{aligned}
& \propto(\eta)=\left(5 \gamma_{0}-\frac{T_{c}{ }^{\prime}}{T_{c}}+2 \frac{\nu_{c}{ }^{\prime}}{T_{c}}\right) \bar{\Theta}_{W}{ }^{(0)}(\eta), \\
& \Theta(\eta)=\bar{\theta}_{T}(\eta), \\
& \mathcal{C}(\eta)=-\left(5 \gamma_{0}-\frac{T_{c}{ }^{\prime}}{T_{c}}+2 \frac{\nu_{c}{ }^{\prime}}{\nu_{v}}\right) c_{M}(\eta)+c_{T}(\eta),
\end{aligned}
$$

where $c_{M}(\eta)$ and $c_{T}(\eta)$ are given by Eqs. (5.6) and (5.12).

Although some of the coefficients in the uniform expansions for $\theta(\eta)$ have, for reasons of simplicity, been obtained by matching, it can be shown, by a straight-forward but somewhat lengthy calculation, that they do, in fact, satisfy the differential equations referred to in the discussion at the beginning of this section. Furthermore, the corresponding coefficients in the expansions for $\Phi(\eta), F(\eta), R(\eta)$, and $\Pi(\eta)$ can be derived from the same set of equations and they are quoted in the appendix.

6. Discussion. The theory developed in this paper provides uniform approximations to the solutions of the Dunn-Lin equations, which are valid in a full neighborhood of the turning point $\eta=0$. Thus far, only "first approximations" have been obtained, but it is clear that by using the present algorithm, higher-order approximations can be systematically derived if required. In that case, the full generality of the generalized Airy functions would be needed.

To the first order, these approximations are strikingly simple, despite the relative complexity of the governing equations, and they can readily be expressed in terms of quantities many of which are well-known from the older heuristic theories. The slowly varying coefficients involve only the regular inviscid solution, the well-balanced part of the singular inviscid solution, and the regularized forms of the first two terms of the Poincare series in the WKB-type outer expansions. On the other hand, the rapidly varying parts consist of only the generalized Airy functions. The inner and outer expansions of the Dunn-Lin equations can, of course, be obtained by re-expansion of these uniform approximations.

No claim of generality has been made throughout the present work. It is expected, however, that the techniques developed here can be easily adapted to deal with the more complicated stability equations of compressible boundary layers. It should be noted, 
however, that the present theory depends crucially on the fact that the inner expansions of Eqs. (2.9) can be expressed in terms of the generalized Airy functions. For the case where the Prandtl number differs from unity, a more general class of functions will be needed.

The derivation of the characteristic equation and the detailed calculation of the curves of neutral stability have not been considered in the present paper mainly because the numerical work involved will itself be a major undertaking. Moreover, such a task is more appropriate as a sequel to the corresponding calculation in the incompressible case.

Appendix. The uniform approximations to $\Phi, F, R$ and $\Pi$. The slowly varying coefficients in the uniform approximations to $\Theta(\eta)$ have been derived in Sec. 5 by the use of the inviscid equations and the method of matching. In this appendix, we shall give the corresponding coefficients in the uniform approximations to $\Phi(\eta), F(\eta), R(\eta)$ and $\Pi(\eta)$. For this purpose, it is convenient to augment the notation of Sec. 3 by defining $\mathbf{q}_{, k}=\left(\begin{array}{l}\Phi_{, k} \\ \theta_{, k} \\ F_{, k} \\ R_{, k} \\ \Pi_{, k}\end{array}\right), \quad \mathbf{u}=\left(\begin{array}{l}B_{k}(\zeta ; 1,1) \\ B_{k}(\zeta ; 0,1) \\ B_{k}(\zeta ;-1,1)\end{array}\right), \quad \mathbf{v}=\left(\begin{array}{l}A_{k}(\zeta ; 1) \\ A_{k}(\zeta ; 0) \\ A_{k}(\zeta ;-1)\end{array}\right), \quad \mathbf{w}=\left(\begin{array}{l}\phi_{w}(\eta) \\ \theta_{w}(\eta) \\ f_{w}(\eta) \\ r_{w}(\eta) \\ \boldsymbol{\pi}_{w}(\eta)\end{array}\right)$,

$$
\mathbf{e}=\left(\begin{array}{c}
\epsilon^{3} \\
\epsilon^{2} \\
\epsilon^{2} \\
\epsilon^{2} \\
\epsilon^{3}
\end{array}\right), \quad L=\left(\begin{array}{lll}
\phi_{, 1} & \epsilon^{2} \phi_{, 0} & \epsilon \phi_{,-1} \\
\theta_{, 1} & \epsilon^{-1} \theta_{, 0} & \epsilon \theta_{,-1} \\
f_{, 1} & \epsilon^{-1} f_{, 0} & \epsilon f_{,-1} \\
r_{, 1} & \epsilon^{-1} r_{, 0} & \epsilon r_{,-1} \\
\pi_{, 1} & \epsilon^{2} \pi_{, 0} & \epsilon \pi_{,-1}
\end{array}\right) .
$$

It can then be verifi $d$ that the structure of the uniform approximations of dominantrecessive types can be defined by

$$
\mathbf{q}_{M, k}=L_{M} \mathbf{v}+O(\mathbf{e}), \quad \mathbf{q}_{T, k}=L_{T} \mathbf{v}+O(\mathbf{e}),
$$

and the uniform approximations of balanced type must then satisfy

$$
\mathbf{q}_{B, k}=L_{B} \mathbf{u}+\mathbf{w}(\eta)+0(\mathbf{e}),
$$

where $L_{B}=s_{M} L_{M}+s_{T} L_{T}$, and where $s_{M}$ and $s_{T}$ are the constants given by (5.19). Clearly, in the present notation, the various coefficients in the uniform approximation to $\theta(\eta)$ are given by

$$
\begin{aligned}
& \theta_{M, 1}(\eta)=a_{M}(\eta), \quad \theta_{M, 0}(\eta)=b_{M}(\eta)=0, \quad \theta_{M,-1}(\eta)=c_{M}(\eta), \\
& \theta_{T, 1}(\eta)=a_{T}(\eta)=0, \quad \theta_{T, 0}(\eta)=b_{T}(\eta), \quad \theta_{T,-1}(\eta)=c_{T}(\eta), \\
& \theta_{B, 1}(\eta)=a(\eta), \quad \theta_{B, 0}(\eta)=\mathbb{B}(\eta), \quad \theta_{B,-1}(\eta)=\mathfrak{C}(\eta), \quad \theta_{w}(\eta)=\mathcal{G}(\eta) .
\end{aligned}
$$

Moreover, in giving the corresponding results for $\Phi(\eta), F(\eta), R(\eta)$, and $\Pi(\eta)$, we shall suppose that the regular inviscid solutions $\Phi_{w}{ }^{(0)}(\eta), \bar{F}_{w}{ }^{(0)}(\eta), \bar{R}_{w}{ }^{(0)}(\eta)$ and $\Pi_{w}{ }^{(0)}(\eta)$ have 
been determined by solving the inviscid equations and their normalizations fixed in terms of that of $\Phi_{w}{ }^{(0)}(\eta)$ (cf. Sec. 4). The coefficients in the uniform approximations to $\Phi(\eta), F(\eta), R(\eta)$, and $\Pi(\eta)$, except for the well-balanced parts of the balanced type approximations, can then be determined algebraically and directly without further matching from the set of equations which they satisfy.

The dominant-recessive approximations of M-type.

$$
\begin{gathered}
\phi_{M, 1}=-\Phi_{w}{ }^{(0)}, \\
\phi_{M, 0}=-\frac{1}{g_{4}}\left[2 \theta_{M, 1^{\prime}}+\gamma \theta_{M, 1}+2 \eta \theta_{M,-1}+(1+\gamma \eta) \theta_{M,-1}{ }^{\prime}+g_{2} \eta \pi_{M, 0}\right], \\
\phi_{M,-1}=\frac{\Phi_{w}{ }^{(0)}}{\eta} . \\
f_{M, 1}=-\bar{F}_{w}{ }^{(0)}, \quad f_{M, 0}=0 \\
f_{M,-1}=i\left[\frac{g_{6} \eta}{\rho} r_{M,-1}+\eta^{\prime} \phi_{M, 0}+\frac{\rho^{\prime}}{\rho} \phi_{M,-1}+\eta^{\prime} \phi_{M,-1}{ }^{\prime}\right] . \\
r_{M, 1}=\bar{R}_{w}{ }^{(0)}, \quad r_{M, 0}=0, \quad r_{M,-1}=\rho\left[\pi_{M,-1}-\frac{\theta_{M,-1}}{T}\right] . \\
\pi_{M, 1}=-\Pi_{w}{ }^{(0)}, \quad \pi_{M, 0}=\frac{1}{g_{3}} \phi_{M, 1}-\pi_{M,-1}{ }^{\prime}, \quad \pi_{M,-1}=\frac{\Pi_{w}{ }^{(0)}}{\eta} .
\end{gathered}
$$

The dominant-recessive approximations of T-type.

$$
\begin{gathered}
\phi_{T, 1}=0, \quad \phi_{T, 0}=-\frac{1}{g_{4}}\left[\theta_{T, 0^{\prime}}{ }^{\prime \prime}+\gamma \theta_{T, 0^{\prime}}+2 \eta \theta_{T,-1}{ }^{\prime}+(1+\gamma \eta) \theta_{T,-1}\right], \\
\phi_{T,-1}=-\frac{1}{g_{4}}\left[2 \theta_{T, 0^{\prime}}+\gamma \theta_{T, 0}\right] . \\
f_{T, 1}=0, \quad f_{T, 0}=i \frac{g_{6} \eta}{T}\left[2 \theta_{T, 0^{\prime}}+\gamma \theta_{T, 0}-\frac{T^{\prime}}{T \eta^{\prime}} \theta_{T, 0}\right] \\
f_{T,-1}=i\left[\frac{g_{6} \eta}{\rho} r_{T,-1}+\eta^{\prime} \phi_{T, 0}+\frac{\rho^{\prime}}{\rho} \phi_{T,-1}+\eta^{\prime} \phi_{T,-1}{ }^{\prime}\right] \\
r_{T, 1}=0, \quad r_{T, 0}=-\frac{\rho}{T} \theta_{T, 0}, \quad r_{T,-1}=-\frac{\rho}{T} \theta_{T,-1} . \\
\pi_{T, 1}=0, \quad \pi_{T, 0}=0, \quad \pi_{T,-1}=0 .
\end{gathered}
$$

The uniform approximations of balanced type. The various coefficients of $B_{k}(\zeta ; p, 1)(p=1,0,-1)$ in the approximations of balanced type can be obtained as linear combinations of the corresponding coefficients in the approximations of dominant-recessive types of using Eq. (A3) and hence their detailed forms will be omitted for brevity.

The well-balanced parts $\phi_{w}(\eta), \theta_{w}(\eta), f_{w}(\eta)$, and $r_{w}(\eta)$ of $\Phi_{B, k}, F_{B, k}$, and $\Pi_{B, k}$ cannot however, be readily expressed algebraically in terms of the well-balanced part $\theta_{w}$ of 
$\Theta_{B, k}{ }^{4}$ They can be obtained, however, either by solving a certain inhomogeneous version of the inviscid equations which they satisfy or, more simply, by the application of the matching method discussed in Sec. 5 , in which case, we have

$$
\begin{aligned}
& \phi_{w}(\eta)=\bar{\phi}_{B}(\eta)+\left(5 \gamma_{0}-\frac{T_{c}{ }^{\prime}}{T_{c}}+2 \frac{\nu_{c}{ }^{\prime}}{\nu_{c}}\right)[\log \epsilon-\gamma+2 \pi i] \Phi_{w}{ }^{(0)}, \\
& f_{w}(\eta)=\bar{f}_{B}(\eta)-\frac{f_{T, 0}(\eta)}{\eta}+\left(5 \gamma_{0}-\frac{T_{c}{ }^{\prime}}{T_{c}}+2 \frac{\nu_{c}{ }^{\prime}}{\nu_{c}}\right)[\log \epsilon-\gamma+2 \pi i] \bar{F}_{w}{ }^{(0)}, \\
& r_{w}(\eta)=\bar{r}_{B}(\eta)-\frac{r_{T, 0}(\eta)}{\eta}+\left(5 \gamma_{0}-\frac{T_{c}{ }^{\prime}}{T_{c}}+2 \frac{\nu_{c}{ }^{\prime}}{\nu_{c}}\right)[\log \epsilon-\gamma+2 \pi i] \bar{R}_{w}{ }^{(0)}, \\
& \pi_{w}(\eta)=\bar{\pi}_{B}(\eta)+\left(5 \gamma_{0}-\frac{T_{c}{ }^{\prime}}{T_{c}}+2 \frac{\nu_{c}{ }^{\prime}}{\nu_{c}}\right)[\log \epsilon-\gamma+2 \pi i] \bar{\Pi}_{w}{ }^{(0)},
\end{aligned}
$$

where $\bar{\phi}_{B}(\eta), \bar{f}_{B}(\eta), \bar{r}_{B}(\eta)$, and $\bar{\pi}_{B}(\eta)$ are the well-balanced parts of the singular inviscid solutions $\bar{\Phi}_{B}^{(0)}(\eta), \bar{F}_{B}{ }^{(0)}(\eta), \bar{R}_{B}{ }^{(0)}(\eta)$, and $\bar{\Pi}_{B}{ }^{(0)}(\eta)$, respectively. It can easily be verified that $\phi_{w}(\eta), f_{w}(\eta), r_{w}(\eta)$, and $\pi_{w}(\eta)$ are regular at $\eta=0$, and that they satisfy the required set of differential equations.

\section{REFERENCES}

[1] D. W. Dunn, On the theory of the stability of the boundary-layer in a compressible fluid, Laboratory Memorandum AE-116, National Aeronautical Establishment, Ottawa, Canada, 1958

[2] D. W. Dunn and C. C. Lin, On the stability of the laminar boundary layer in a compressible fluid, J. Aero. Sci. 22, 4jう̄-477 (195j)

[3] T. H. Hughes and W. H. Reid, The stability of spiral flow between rotating cylinders, Phil. Trans. Roy. Soc. Lond. A263, 57-91 (1968)

[4] L. Lees and C. C. Lin, Investigation of the stability of laminar boundary layer in a compressible fluid, Nat. Adv. Comm. Aero., Wash. TN-1115 (1946)

[5] L. Lees and E. Reshotko, Stability of the compressible laminar boundary layer, J. Fluid Mech. 12, $555-590$ (1962)

[6] C. C. Lin, The theory of hydrodynamic stability, Cambridge University Press, $195 \overline{5}$

[7] C. C. Lin, in Asymptotic solutions of differential equations and their applications (C. H. Wilcox, ed.), John Wiley and Sons, New York, 1964

[8] L. M. Mack, Boundary-layer stability theory, Notes for the A1AA professional study series-highspeed boundary-layer stability and transition, JPL, California Institute of Technology, 1969

[9] F. W. J. Olver, The asymptotic solution of linear differential equations of the second order for large values of a parameter, Phil. Trans. Roy. Soc. Lond. A247, 307-327 (1954)

[10] W. H. Reid, in Basic developments in fluid dynamics (M. Holt, ed.), Academic Press, New York, 1965

[11] W. H. Reid, Composite approximations to the solutions of the Orr-Sommerfeld equation, Studies in Appl. Math. 51, 341-367 (1972)

[12] W. H. Reid, Uniform asymptotic approximations to the solutions of the Orr-Sommerfeld equation, part I: plane Couette flow, Studies in Appl. Math. 53, 91-110 (1974)

[13] W. H. Reid, Uniform asymptotic approximations to the solutions of the Orr-Sommerfeld equation, part II: the general theory, Studies in Appl. Math. 53, 217-224 (1974)

[14] S. F. Shen, in Theory of laminar flows (K. F. Moore, ed.), Princeton University Press, 1964

[15] H. B. Squire, On the stability for three-dimensional disturbances of viscous fluid flow between parallel walls, Proc. Roy. Soc. Lond. A142, 621-628 (1933)

${ }^{4}$ This is due to the fact that the set of differential equations satisfied by $\phi_{w}, f_{w}, \theta_{w}, r_{w}$, and $\pi_{w}$ can be uncoupled and rewritten as a single second-order equation in terms of either $\phi_{w}$ or $\pi_{w}$, but not in terms of $\theta_{w}$. 